International Journal of Engineering \& Technology, $7(1)(2018) 204-209$
International Journal of Engineering \& Technology
SPC
Website: www. sciencepubco.com/index.php/IJET
doi: $10.14419 /$ ijet. $v 7$ il. 8643
Research paper

\title{
Skin cancer detection and stage prediction using image processing techniques
}

\author{
Sheeju Diana $\mathrm{B}^{1}$ *, Ramamurthy $\mathrm{B}^{2}$ \\ ${ }^{1}$ PG Research Scholar, Department of Computer Science, Christ (Deemed to be University), Bengaluru \\ ${ }^{2}$ Associate Professor, Department of Computer Science, Christ (Deemed to be University), Bengaluru \\ *Corresponding author E-mail: Sheeju.b@cs.christuniversity.in
}

\begin{abstract}
Skin cancer is one of the perilous forms of cancer that most recently occurred in preceding and in recent years as well. Early detection of skin cancer is curable and it eliminates the cost that is spent on the advanced treatment. Skin cancer mainly occurs due to exposure to sun's ultraviolet radiation and other environmental threats. It can be categorized into, Melanoma and Non-Melanoma. Melanoma is dangerous one. Once it is occurred it starts spreading across other parts of the body if not treated in the early stages. Non-Melanoma is a static cancer which does not affect the normal cells of the skin. This paper aims to develop an application to detect skin cancer and stage prediction using Image Processing Techniques. Stage is predicted, so that the treatment for the same is done without any delay. Skin cancer affected image is taken as input and various preprocessing techniques is applied for the same. The Preprocessing Techniques such as Noise Removal is applied on the image to filter out the noise. Filtered image is enhanced using Histogram Equalization and image is segmented to extract the affected portion. The Area, Perimeter and Eccentricity values are calculated for the affected portion of the skin. The values are then fed into the Neural Networks using Back Propagation algorithm in order to predict the Stage and type of the Skin cancer.
\end{abstract}

Keywords: Back Propagation; Feature Extraction; Histogram Equalization; Melanoma; Neural Network; Non-Melanoma; Noise Removal; Segmentation.

\section{Introduction}

The functionality of skin plays a vital role in the human body since it is the largest organ which covers the muscles, bones and other parts of the body. Once the functionality of skin goes wrong it affects the other parts of the body [1]. Skin is the most sensitive part, therefore when it is explored into sunlight and other environmental pollution tends to occur skin cancer. Skin cancer appears to be of two kinds Benign and Melanoma form. Benign it's just the moles on the skin which does not penetrate inside, whereas Melanoma causes sores on the skin which leads to bleeding and it is named after cells Melanocytes which is more hazardous [2]. In United States, more than 700,000 skin lesions are diagnosed annually under the estimation of American Cancer Society [3]. According to statistics given by the Apollo and other hospitals it suggests that Melanoma affects the ages ranging from 41-60+. There are technologies that are used to detect skin cancer at the early stages. Skin Cancer detected in advance can save people's lives and it eliminates the multiplication of cancer cells across the parts of the body. Although it affects the people within age limits but high probably is for the bright skin people.

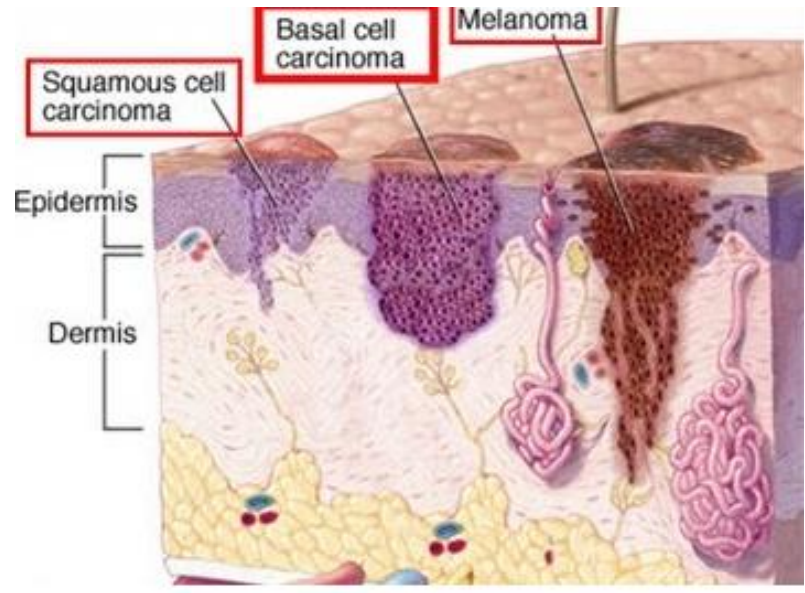

Fig. 1: Different Types of Skin Cancer.

Fig 1 states the different types of skin cancer, the diagram clearly shows the Melanoma penetration inside the skin. [4]

It will be hard for even the experienced dermatologist to detect the skin cancer or to predict the stages. Therefore, many hardware \& software devices and applications evolved. In order to detect NonMelanoma Skin Cancer (NMSC) there are many hardware devices which includes, biopsy, molecular markers, ultra-sonography, Doppler, optical coherence tomography, Dermoscopy and spectroscopy [5]. Dermoscopy is one such hardware device that helps in examining the surface of the skin using skin surface microscopy for the detection of skin cancer; it is a non-invasive examination technique that lends the support to distinguish between Melanoma 
and Non-Melanoma. It is the most expensive device that cannot be affordable for the poor countries and developing countries like Africa. Moreover, later researchers came up with many software applications, which eliminated the hardware cost and influence the ease of usage. The software does not require any expertise to handle them but developed with the intention of prior information about the cancer.

Image Processing is one of the traditional approaches which deals with analyzing and processing of an image. Image processing has proposed many methodologies which helps in the early detection of skin cancer. Proposed methodologies have influenced in the early detection which prevents the cancer from spreading across the skin. The methodology in an image processing approach involves the use of Noise removal, Edge detection, Image enhancement, Segmentation algorithms, Feature selection, Feature extraction, calculating Area, Perimeter, Eccentricity, Neural network approach using Back propagation algorithms. In this paper, a model is proposed with an aim of curing the skin cancer along with providing cancer information to the people. Model detects the skin cancer on the color skin image and uses pre-processing methods such as Image acquisition, Noise removal and plotting Histogram. Pre-processing methods helps to solve the illumination, contrast and noise problems. After the removal of noise various techniques such as Edge detection, Image enhancement, Segmentation. Feature extraction is used to extract the affected portion of skin, then the Area, Perimeter and Eccentricity is calculated. Calculated values are fed into the Neural networks, using Back propagation algorithm the stage and type of the skin cancer is predicted.

The main aim of the proposed system is to eliminate the risk caused by many countries with respect to the skin cancer. Many hardware devices were developed but those devices were not affordable. Patients are not only provided with the information of type of cancer but appropriate stage of the skin cancer is predicted which helps to easily cure the cancer when stage is obtained.

\section{Related works}

Authors used the mapping of intensity values to improvise the skin lesion boundaries are the source of this work. Intensity Thresholding is used for the segmenting of the intensity image which in turn refines the image edges using segmentation. Segmentation technique uses 3 parameters, Gaussian function, Image gradients and sharpness of Colour changes. [6]

The skin lesion image analysis with the consideration of $\mathrm{ABCD}$ (Asymmetry, Border, Colour, Diameter) features using the texture, size and shape for the image segmentation and image feature stages. The extracted features help in distinguishing between a normal skin and a melanoma cancer lesion. [7]

Dermoscopy of the skin image is segmented using Thresholding and features of a cancer cell are extracted using 2D wavelet transform method. Feature extraction helps to distinguish between a malignant and Benign melanoma. Back-propagation neural network uses the features as an input node and classifies the given dataset into cancerous and non-cancerous. Methods provided an acceptable accuracy using the image processing techniques and classifiers. [2]

Segmentation and other methods are used for the automatic initialization of seed points. The skin lesion areas are identified using the Colour and texture features with the relative applications of common classifiers such as SVM \& KNN. The combination of these classifiers can increase the accuracy of the model. The classifiers such as ANN \& PNN are more accurate than SVM \& KNN with high accuracy in feature $\&$ decision levels. [1]

Region-based segmentation with some isodata algorithm determines the threshold for detection. Neural network detection and rational Gaussian provides a framework for recognizing the network edge patterns. The results of IS and ASS methods provide the lesion boundary with some curve fitting techniques. IS method for the skin cancer detection has given more accuracy than the other methods which were used for skin cancer diagnosis. [8] Segmentation techniques such as threshold based and edge based has helped to identify the boundaries of the skin lesions. Feature extraction processes the acquired boundary and uses the ABCD (Asymmetry, Border, Colour and Diameter) features to identify the affected part of the skin. TDS calculation defines a threshold which distinguishes between the skin cancerous image and noncancerous image. The system is more reliable and interactive for the users [9].

Unsupervised segmentation techniques are used for the analysis of the digital images. Feature extraction techniques are applied on the segmented images with the segmentation methods such as Otsu's method, Gradient vector flow and colour based image segmentation. Colour variance measurements are calculated for the closeness between different colours. Diameter measurement is used for the calculation of melanoma of the diameter of $6 \mathrm{~mm}$. It provides an awareness for the occurrence of skin cancer [10].

Neural network is used for establishing the relationship of skin cancer image with an automatically skin cancer classification system. Karhunenloeve is a method which transforms histogram equalization into different stages of cancer. Region of Interest (ROI) with the help of segmented used to extract the important feature of the skin lesion. Back Propagation and Auto associative neural network algorithms are used for the cancer detection \& works with an accuracy of $80 \%$ [11].

Multilayer perceptron classifier (MLP) was proposed for the training and testing phase. MLP helped to distinguish between Malignant Melanoma and melanocytic nevi. Texture analysis were used for the calculation of occurrence of the Melanocytic nevi which contained 23 features out of which 12 were selected as main features. Automatic and traditional MLP are comparatively slower compared to the ANN classifier [12].

Features of the skin are extracted using the techniques such as segmentation, border detection and texture processing. Classification methods such as SVM and CART were used with the Bayesian networks. The model proposed in the paper eliminates the usage of ELM and video microscopy which involves a high noise. SVM influences the performance of the cancer detection. Application of CDSS system in the detection of cancer characterization is affordable. [13].

Image enhancement a technique to reduce the noise, scratches and occlusions. Feature extraction helps in the extraction of the lung affected regions, where the calculation such as area $\&$ perimeter is calculated. The area \& perimeter is calculated to validate the spread of the lung cancer across the cells. Classification algorithms are used to define the stages of occurrence of the lung cancer [14].

Magnetic Resource Images (MRI) are processed using the Unsupervised Neural Network Algorithm. The segmentation of MRI image is quite difficult but the segmentation can be done using the UNN algorithms. The Proposed algorithm influence the accuracy in deriving output as well as provides inputs to make the network ready for the processing of MRI images. The algorithm takes much time to train itself. [15].

Various images in painting methods are used for the filling of the unknown regions. The affected area of the skin is examined using the methods such as Fuzzy C-Means (FCM) and Markov Random Field (MRF). The application of binary image is used to map the original image for the detection purpose. Gabor or in painting filters are used in the Dermoscopy image of the skin to eliminate the noise, scratches and hairs from the skin. [16]. 


\section{Proposed system}

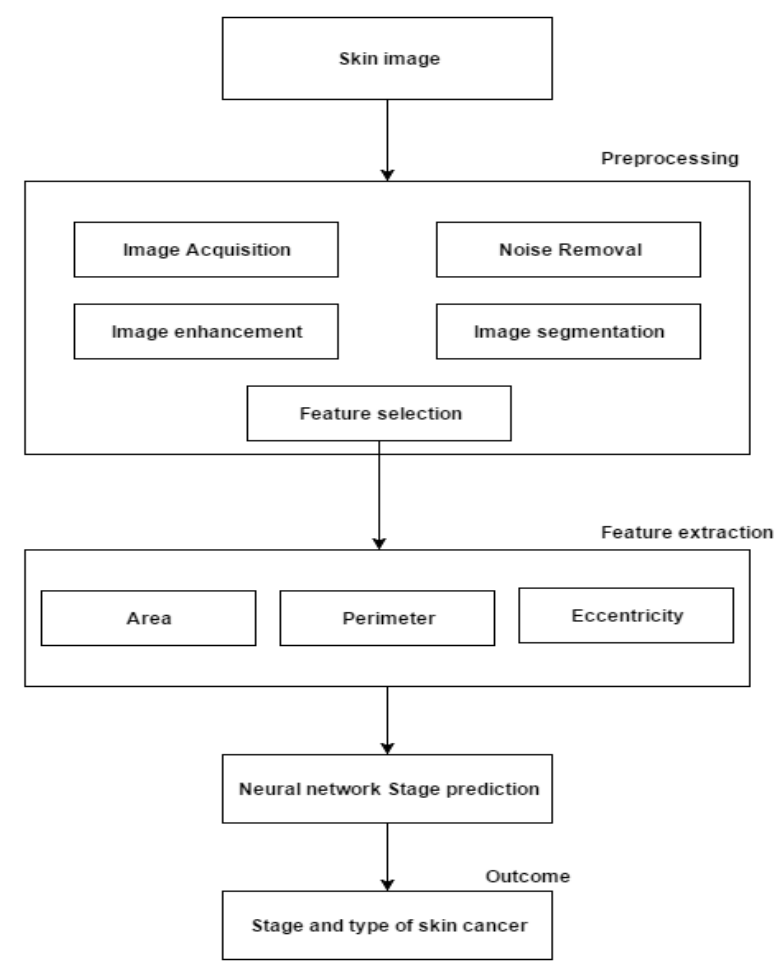

Fig. 2: Proposed System.

Image processing is used across many areas, one among them is the medical field. Detection of skin cancer using the image processing techniques provides precautionary measures and gives the accurate information to the patients. Proposed system is a skin cancer detection and prediction system which is developed using Image Processing Techniques. Affected skin image is taken as an input into the proposed system and the image is converted to grayscale image with which many functions can be applied. Once the Image acquisition is achieved pre-processing techniques are implemented using the Noise removal algorithms. After the noise removal, the image is enhanced using Histogram equalization to enhance the contrast \& quality of the image. Histogram is also plotted to know the peak ratio of the affected pixels. Edge detection highlights the edges of the affected portion which helps to get an idea of where the cancer is majorly affected. Segmentation algorithms are implemented in order to threshold the image, so that the effected portion and unaffected portion is differentiated by darkening the majorly infected portion. Features are finally extracted to find the Area, Perimeter, Eccentricity, the calculated values are fed into the Neural Networks using Back Propagation algorithm and activation functions which helps in predicting stage and type of skin cancer. The Techniques used in the model are given below:

\subsection{Image acquisition}

Skin image is taken as an input from the patient through the proposed system. System is developed in such a way that it can accept only colour images as its input. In fig 3 and 3.1 the colour image is then converted to grayscale, which is a basic operation

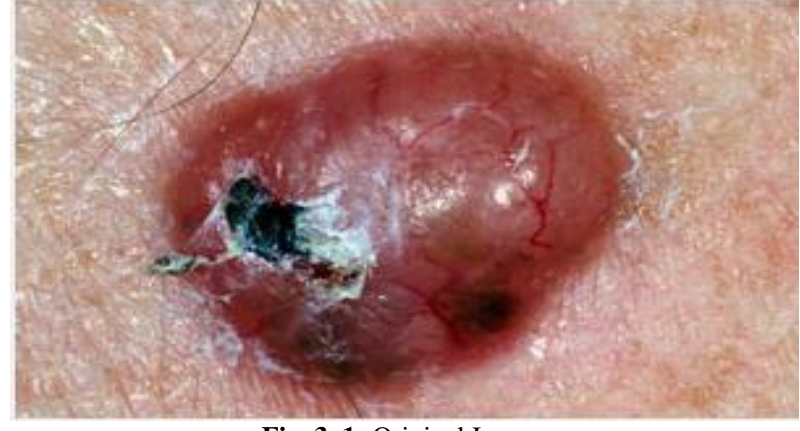

Fig. 3. 1: Original Image.

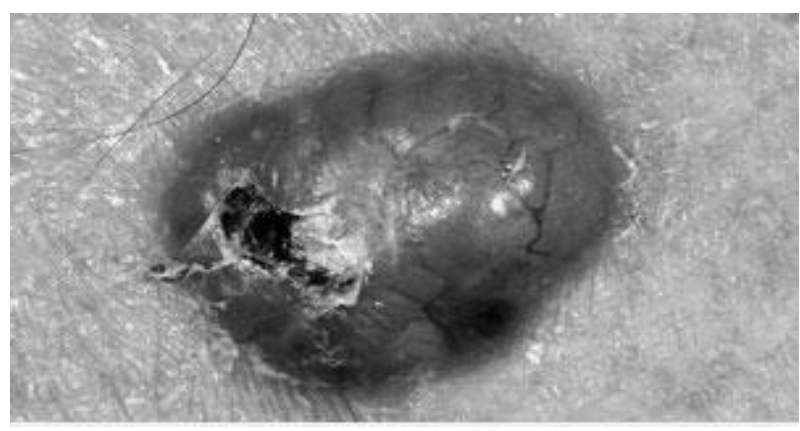

Fig. 3. 2: Grayscale Image.

Fig 3 Conversion from RGB to gray scale.

\subsection{Noise removal}

Medical images are prone to much noises. Noise can be called as irrelevant information that surrounds the image in the current domain. Obtained skin image is prone to much noise; these noises are removed using the Filter techniques. One of them is Median filter which eliminates the additional noise from the skin image and smoothens the quality of the image.

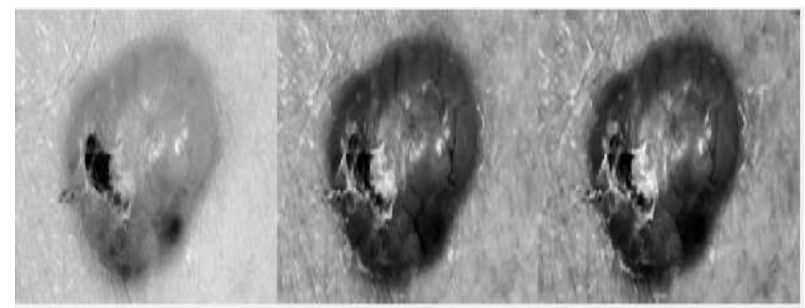

Fig. 4: Noise Removed Image.

Input image is added with a little noise to make it better for smoothening. Applies median filter on the pixels of the input image, which results in the process given in Fig 4. The outcome of the noise removal process is a smoothened image.

\subsection{Image enhancement}

The outcome of the Noise removal is the filtered image which is given as an input to stretch its quality and enhance its contrast that can be used based on the context of the domain. By enhancing the skin image clarity of the image increases more than the processed image. Fig 5 is an example for Image Enhancement.

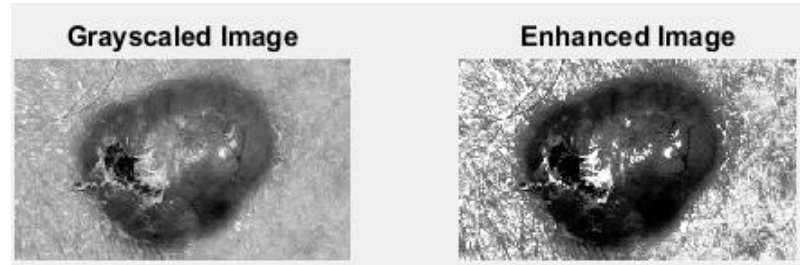

Fig. 5: Image Is Enhanced from Grayscale to High Contrast Image. 


\subsection{Edge detection}

As shown in Fig 6, Edges of the affected skin are detected using the canny edge detector and applies binarization over the image to highlight into a black \& white image to detect the affected portion.

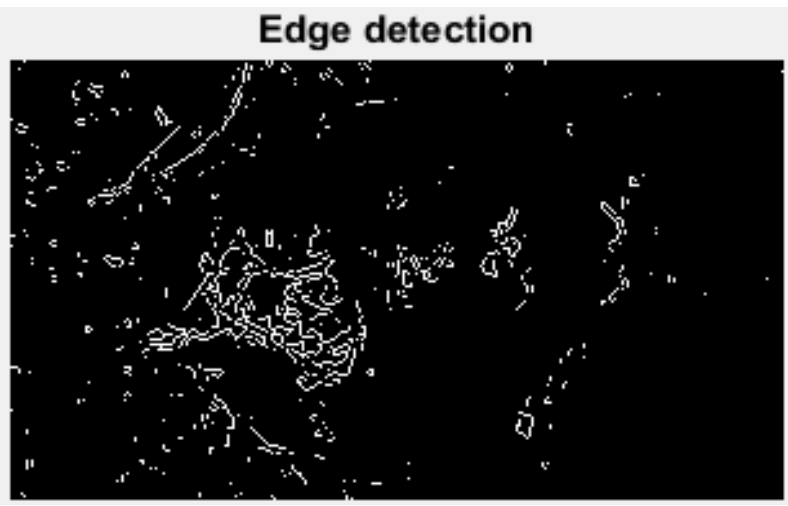

Fig. 6: Edge Detected Image.

\subsection{Plotting histogram}

To estimate the range of affected pixels' histogram is plotted, so that majorly infected portion can be easily determined.

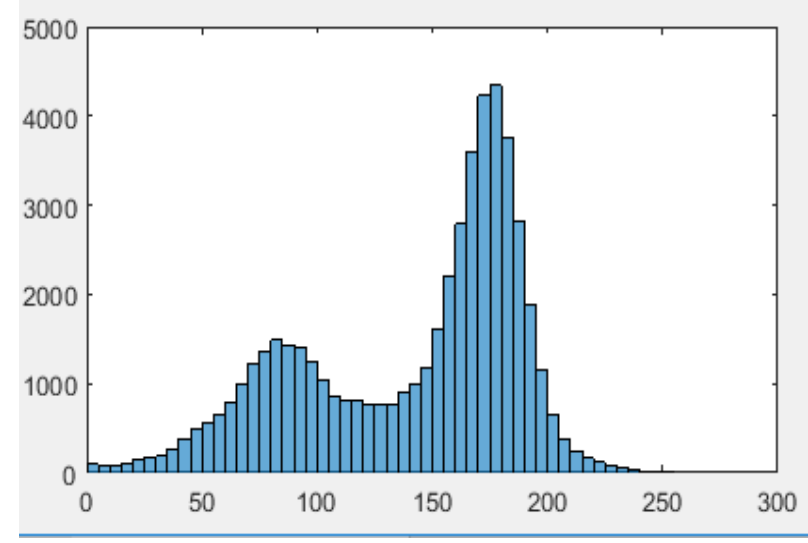

Fig. 7: Histogram Representation.

\subsection{Feature selection}

Enhanced image based on the domain is fed into the feature selection algorithms, where the proposed system follows the colour based feature selection model. Processed image's colour such as red, brown or pink is selected and that part of the image is highlighted \& used for the further process. Fig 8 exhibits the feature selection.

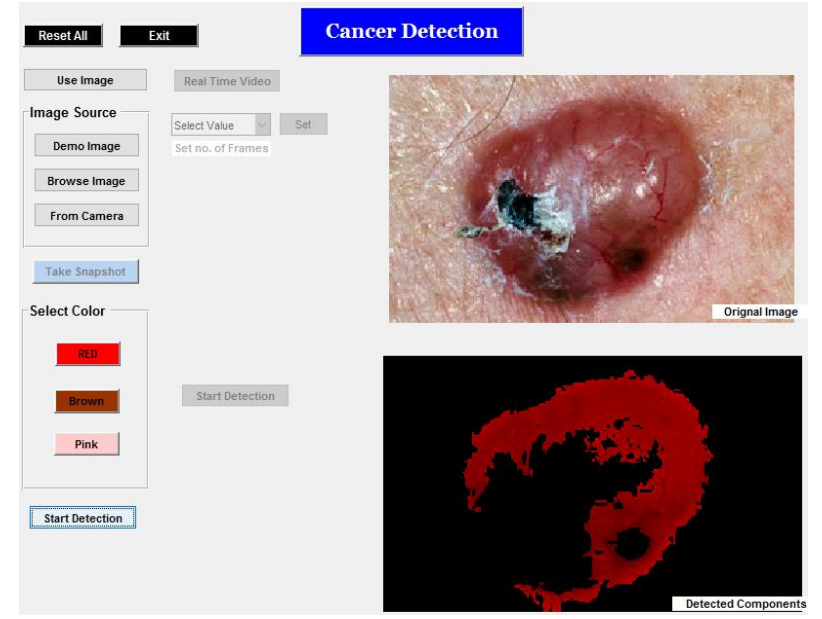

Fig. 8: Feature Selection.

\subsection{Segmentation}

The first level of segmentation is Thresholding. Thresholding value greater than 170 is evaluated for all the channels of the input image and converts the output into a binary image for better system evaluation. Uses the individual channels to map the affected portion on the top of the binary image in order to highlight only the affected portion.

Uses binarization method to convert the image into binary, that can help in highlighting the affected portion much easily.

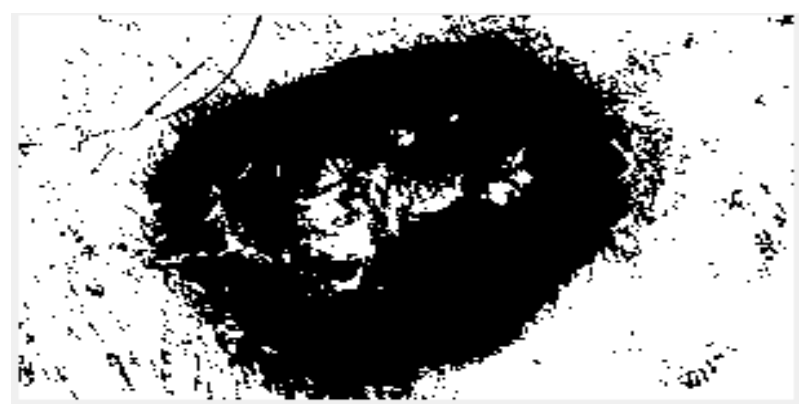

Fig. 9.1: Affected Skin Background Removal.

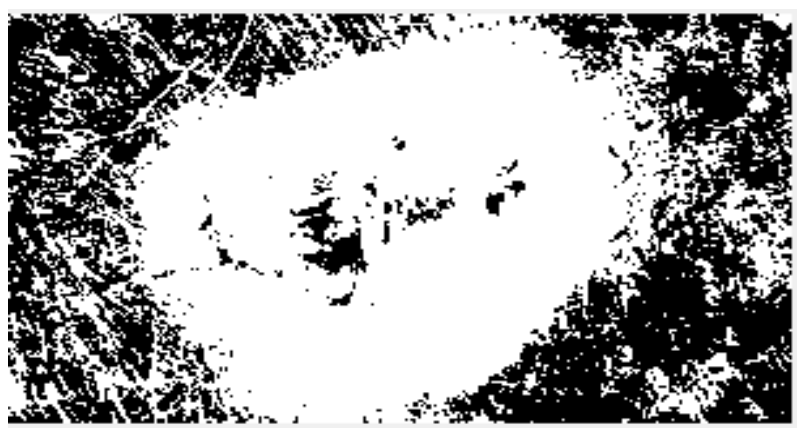

Fig. 9.2: Affected Region Removal.

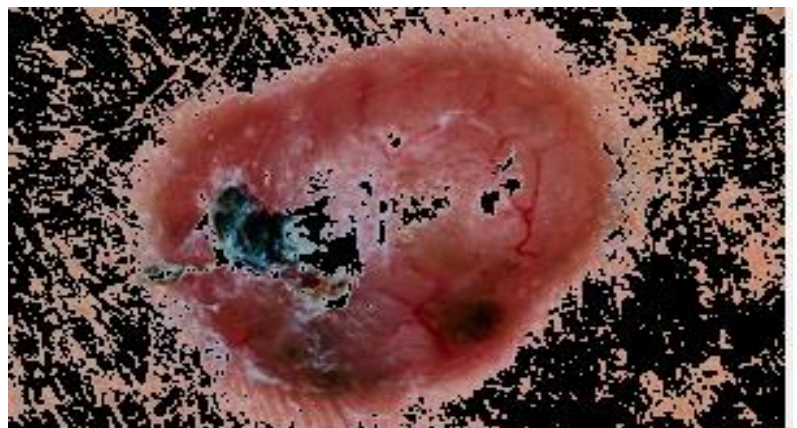

Fig. 9.3: Mapping the Affected Region on the Original Background

Fig 9 The outcome of Segmentation based on Thresholding approach.

\subsection{Feature extraction}

Output of the segmented skin image is considered as the input and important skin characters for the input is processed. Skin characters include calculation of Area, Perimeter and eccentricity.

1) AREA - important skin character that is calculated for the affected skin portion. It is determined by the formula:

$A=\Sigma_{i} \Sigma_{j}\left(A_{i j}, X-R O I[A r]=i, Y-R O I[A r]=j\right)$

Where $i, j$ - pixels within the shape.

ROI- region of interest.

$\mathrm{X}$ - ROI [] - vector contain ROI x position

$\mathrm{Y}$ - ROI [] - vector contain ROI y position

2) PERIMETER - determines the depth of the skin cancer affected image and it is determined by the formula: 
$\mathrm{B}=\Sigma_{\mathrm{i}} \Sigma_{\mathrm{j}}\left(\mathrm{P}_{\mathrm{ij}}, \mathrm{X}-\operatorname{edge}[\mathrm{P}]=\mathrm{i}, \mathrm{Y}-\operatorname{edge}[\mathrm{P}]=\mathrm{j}\right)$

X_edge [] - vector representing coordinate of ith pixel.

Y_edge [] - vector representing coordinate of jth pixel

3) ECCENTRICITY - is a calculation done by considering the max and min value of the affected skin image and it is determined by the formula:

$\mathrm{E}=$ Length of major axis / Length of minor axis

\subsection{Stage prediction using back propagation neural network}

Neural network also called as Artificial Neural Networks which was influenced by the biological neural networks. It is most commonly used in the information processing systems and each of the neural networks has 3 layers: Input layer, Hidden layer and Output layer. These layers undergo two process, namely: Training, Validation and Testing. Training is a process of adjusting the weights of the network, Validation deals with the adjustment of the parameters and testing deals with the prediction of the outcome.

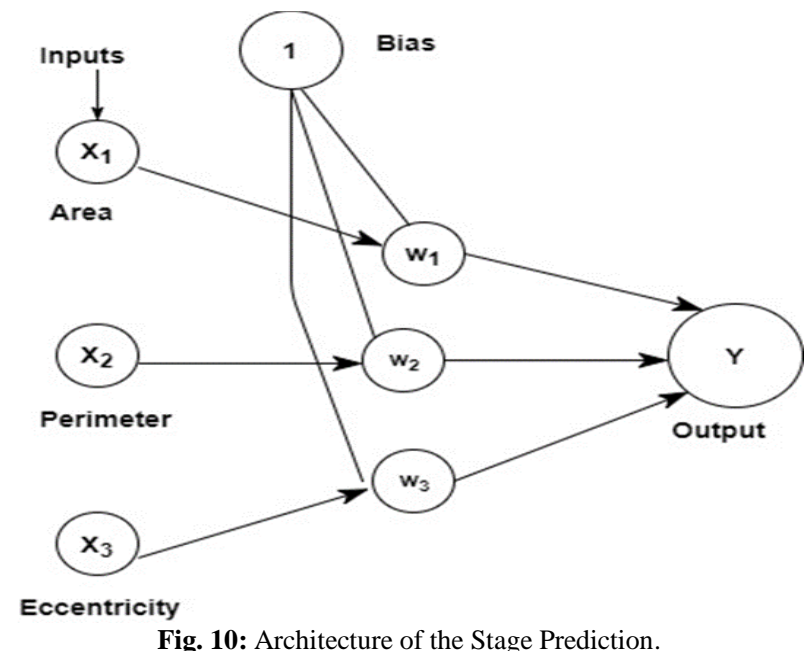

The Architecture of BPN is shown in figure 10. Proposed system uses the Backpropagation for the prediction of the stage of skin cancer. The outcomes of the feature extraction, namely: Area, Perimeter and Eccentricity is fed as an input to the Backpropagation Neural network. Each of these inputs are multiplied with the weights of the network, weights are user defined based on the particular domain of the application. W1, W2 and $\mathrm{W} 3$ values are considered to determine the weighting factor, each of the $\mathrm{W}$ values can range from 0-1 and the biased function is added with each of the multiplied values, that is 1 .

$($ Area*W1+b)+(Perimeter*W2+b) $+($ Eccentricity*W3+b) $=\mathrm{Y}$

Employs activation function such as RAMP to the output layer in order to predict the stage of the cancer as shown in figure:

$$
\mathrm{f}(x)=\left\{\begin{array}{lr}
1 & \text { if } x>1 \\
x & \text { if } 0 \leq x \leq 1 \\
0 & \text { if } x<0
\end{array}\right.
$$

Ramp function is responsible for the stage prediction and its range is shown in figure 11. If $\mathrm{f}(\mathrm{x})>1$ then the skin lesion belongs to stage 3 that is dangerous, if $\mathrm{f}(\mathrm{x})<=0 \& \mathrm{f}(\mathrm{x})<=1$ then the skin lesion belongs to the stage 2 and if $\mathrm{f}(\mathrm{x})<0$ then it is considered to be stage 1 . The function considers only the affected portion's area, perimeter and eccentricity, ignores the non-cancer images.

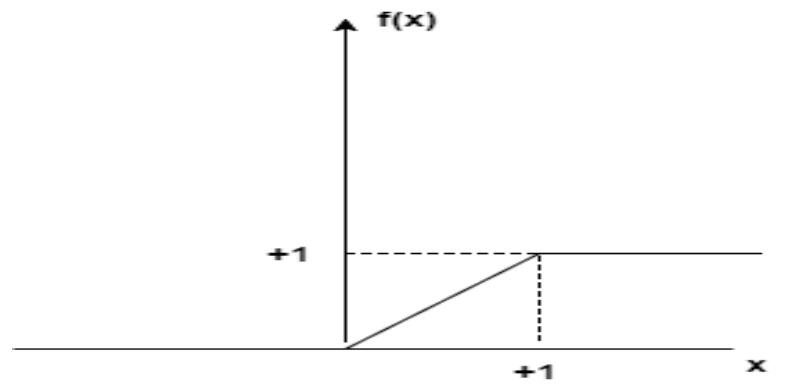

Fig. 11: Ramp Function.

BPN allows to modify the weighting factors to obtain the determined outcome, if the determined outcome is not obtained again weighting factors can be modified to get the expected outcome. This is one of the advantage of applying BPN (Backpropagation Network) on the prediction of skin stage.

\section{Results and discussions}

The proposed system was tested for a ISIC (International Skin Imaging Collaboration) dataset of image dimensions $620 * 250$ pixels. System mainly concentrated on the results of the skin characters such as area, perimeter and eccentricity. Result obtained for 5 images is as follows:

\begin{tabular}{|c|c|c|c|}
\hline IMAGE & A & P & E \\
\hline & & & \\
\hline & 78.57 & 7.814 & 1.601 \\
\hline & 6.761 & 42.73 & 1.796 \\
\hline & 1.273 & 22.36 & 1.978 \\
\hline & & & \\
\hline & & & \\
\hline & & & \\
\hline & & & \\
\hline
\end{tabular}

Fig. 12: Outcome of Feature Extraction Stage Prediction Using BPN. 


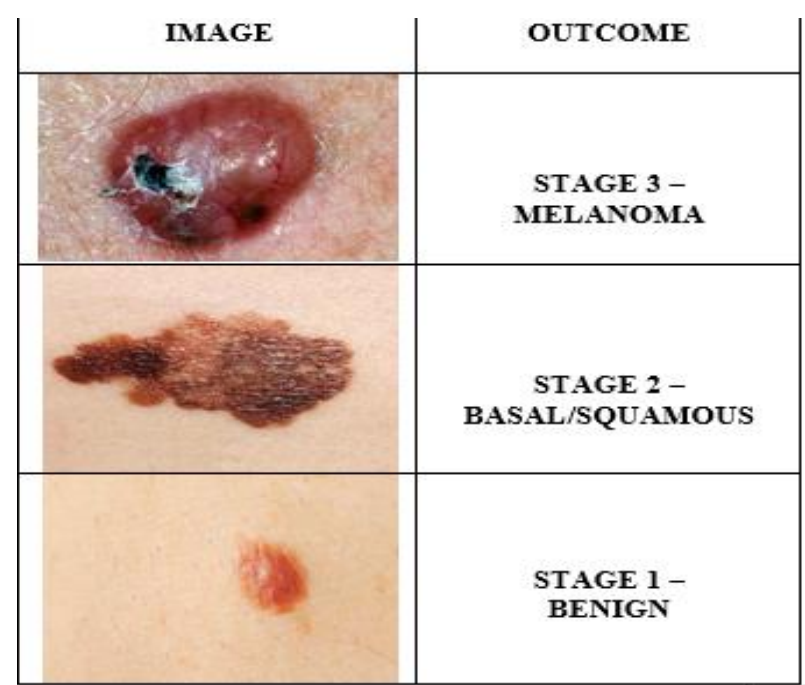

Fig. 13: Outcome of Stage Prediction.

In the above figure 12, A-Area, P-Perimeter and E-Eccentricity. Outcomes of the stage prediction is determined by the Values obtained from the Ramp function of BPN (Back Propagation) When the $f(x)>1$ then it is stage 3 - Melanoma similarly when $\mathrm{f}(\mathrm{x})=0 \& \mathrm{f}(\mathrm{x})<=1$ then it is stage $2-$ Basal and if $\mathrm{f}(\mathrm{x})<0$ then it is stage 1 - Pre-cautionary measures have to be taken. Each of these outcomes are only for the affected skin lesions.

Proposed model deals with the Skin cancer detection and stage prediction that provides the relevant information for the patients, so that the appropriate treatment can be taken. Comparing the proposed model to the "Classification of Lung cancer stages on CT scan images using image processing" authored by Kulkarni, where the base paper deals with the detection of lung cancer and does not employ neural networks for prediction of stages. Proposed system employs Backpropagation Neural Network which provides a major advantage of reproducing the expected outcome based on the weight factors and activation function. Apart from "Classification of Lung cancer stages on CT scan images using image processing" there are various other papers which only deals with the detecting skin cancer, stage of the skin cancer is not predicted. In this paper stage is predicted using BPN which majorly helps poor people. This eliminates the cost that is spent on expensive treatment.

\section{Conclusion}

Skin cancer is one of the dangerous forms of cancer as the affected cells can spread easily across the body. It can be either Melanoma or Non-Melanoma. There are various solutions such as Dermoscopy and other devices to detect the skin cancer, these devices involve costs as well as requires a doctor to equip them on the patients. Proposed method aims at detecting and prediction of skin cancer using Image Processing Techniques that can be easily used by Doctors for the Patient's skin cancer analysis. The system employs methods such as Preprocessing, Feature Selection, Feature Extraction and Backpropagation Neural Networks. The outcome of the model is determined by the BPN (Backpropagation Network) that predicts the type of the cancer.

This kind of models help the patients to take care of their skin as well as take precautionary measures if the Skin cancer is encountered. The model was applied on a ISIC (International Skin Imaging Collaboration) dataset and resulted in the classification of the cancer types.

\section{Acknowledgement}

I Would Like To Take This As An Opportunity To Thank The Almighty And My Parents For Their Love And Support. Further Extend My Thankfulness To The Department Of Computer Sci- ence, Christ (Deemed To Be University) And All The Faculties Who Had Been A Tremendous Support Towards My Completion Of My Research Work

\section{References}

[1] R. Sumithra, M. Suhil and D. Guru, "Segmentation and Classification of Skin Lesions for Disease Diagnosis", Procedia Computer Science, vol. 45, pp. 76-85, 2015 https://doi.org/10.1016/j.procs.2015.03.090.

[2] Jaleel, S. Salim and A. R. B, "Artificial Neural Network based detection of Skin Cancer", International journal of Advanced research in Electronics, Electrical and Instrument engineering, vol. 1, no. 3, 2012.

[3] D.E. Elder, Skin cancer: Melanoma and other specific nonmelanoma skin cancers, Cancer Supplement 75 (1) (1994) 245-256.

[4] http://www.skindermatologists.com/squamous-cell-carcinomascc.html

[5] Salah, B., Alshraideh, M., Beidas, R. and Hayajneh, F. (2011). Skin Cancer Recognition by Using a Neuro-Fuzzy System. Cancer Informatics, 10, p.CIN.S5950. https://doi.org/10.4137/CIN.S5950.

[6] L. Xu, M. Jackowski and A. Goshtasby, "Segmentation of skin cancer images", Image and Vision Computing, vol. 17, no. 1, pp. 65-74, 1999. https://doi.org/10.1016/S0262-8856(98)00091-2.

[7] S. Jain, V. jagtap and N. Pise, "Computer aided Melanoma skin cancer detection using Image processing", in International conference on Intelligent computing, communication and convergence, Bhubaneshwar, India, 2015, pp. 736-741.

[8] M. Rajab, M. Woolfson and S. Morgan, "Application of regionbased segmentation and neural network edge detection to skin lesions", Computerized Medical Imaging and Graphics, vol. 28, no. $1-2$, pp. 61-68, 2004. https://doi.org/10.1016/S08956111(03)00054-5.

[9] S. Jaiswar, M. Kadri and V. Gatty, "Skin cancer detection using Digital image processing", International Journal of scientific engineering and research (IJSER), 2014.

[10] bhuiyan, I. Azad and k. uddin, "Image processing for skin cancer feature extraction", International journal of scientific and Engineering research, vol. 4, no. 2, 2013.

[11] H. Lau and A. Al-jumaily, "Automatically Early detection of skin cancer : Study based on neural network classification", 2009.

[12] M. A sheha, M. Mabrouk and A. Sharawy, "Automatic Detection of Melanoma Skin cancer using Texture Analysis", International Journal of of Computer Application, vol. 42, no. 20, 2012.

[13] Maglogiannis and C. N Doukas, "Overview of Advanced computer visions systems for skin lesions characterization", IEEE transactions of Information technology in Biomedicine, vol. 13, no. 5, 2009. https://doi.org/10.1109/TITB.2009.2017529.

[14] Kulkarni and A. Panditrao, "Classification of Lung cancer stages on CT scan images using image processing", IEEE International conference on Advanced communication control and computing techniques $\quad$ (ICACCCT), 2014 https://doi.org/10.1109/ICACCCT.2014.7019327.

[15] Abdulbaki, "Skin Cancer Image Segmentation \& Detection by using Unsupervised Neural Networks (UNN)", 13th ARAB international conference, 2012

[16] K. Eltayef, Y. Li and X. Li, "Detection of Melanoma skin cancer in Dermascopy images",International conference on Communication, Image and Signal processing, 2016. 